\title{
Review
}

\section{Advances in rice chromosomes research}

\author{
By Nobuko Ohmido, ${ }^{*)}$ Kiichi FukuI, ${ }^{* *)}$ and Toshiro Kinoshita ${ }^{* * *), * * * *), ~ †) ~}$ \\ (Communicated by Eishiro SHIKATA, M. J. A.)
}

\begin{abstract}
Rice chromosome research was initiated around 1910 and has been progressing ever since. Twelve pairs of chromosomes $(2 n=24)$ and a $440 \mathrm{Mb}$ genome, the smallest genome among all main cereal crops, have been identified. According to the recent rice chromosome researches, all chromosomes were well identified over the disadvantages of small chromosome size and quantitative rice chromosomal maps have been developed using chromosome image analysis system (CHIAS). Moreover, agriculturally important genes have been detected using new direct DNA visualization methods with fluorescence in situ hybridization (FISH). This review describes the recent progress in molecular and digital cytogenetics using visualization from a historical perspective, highlighting the future prospects in rice chromosome science.
\end{abstract}

Key words: Chromosome; Oryza sativa L.; chromosome image analysis system (CHIAS); fluorescence in situ hybridization (FISH).

Introduction. Rice, Oryza sativa L., has 24 chromosomes $(n=12)$ and a genome size of $440 \mathrm{Mb}$ at the haploid level. The International Rice Genome Sequencing Project $^{1)}$ was initiated several years ago as a whole genome sequencing project, and the information being gathered is applied for the significant studies of other cereals such as wheat, corn and barley, because of the synteny among these genomes. ${ }^{2)}$ Rice chromosome studies have therefore entered a new era since the latter half of the last century. Especially, new techniques such as chromosome image analysis system (CHIAS) and fluorescence in situ hybridization (FISH) were explored by many cytogeneticists in the 1980's and their application in plant genetics has contributed to produce an abundance of new information. This review describes the history of rice chromosome research mainly from the view point of rice genetics and breeding.

History of rice chromosome research. Due to the importance of rice as a staple food for nearly half the

\footnotetext{
*) Faculty of Human Development, Kobe University, Kobe 657-8501, Japan

**) Department of Biotechnology, Graduate School of Engineering, Osaka University, Suita 565-0871, Japan.

***) Professor Emeritus, Hokkaido University, Sapporo 0608589, Japan

****) Recipient of the Japan Academy Prize in 1993.

$\dagger$ Correspondence to: T. Kinoshita.
}

world's population, cytological researches on rice chromosomes have a long history. The first accurate recording of rice chromosome number $(2 n=2 x=24)$ was published in 1910 based on observations of microsporogenesis, megasporogenesis and mitosis, ${ }^{3)}$ and in 1929, these chromosomes were classified into three types, five large, four medium and three small by Rau. ${ }^{4)}$ Further, Nandi ${ }^{5)}$ reported that rice chromosome size ranges from 0.7 to $2.8 \mu \mathrm{m}$ at mitotic metaphase, while Yasui ${ }^{6)}$ reported a range of 2.0 to $5.0 \mu \mathrm{m}$ for japonica after characterizing all 12 chromosomes using haploid plants by measuring the lengths of mitotic chromosomes. $\mathrm{Hu}^{7)}$ identified seven to eight pairs of different mitotic chromosomes depending on their features due to uneven condensation at the prometaphase stage. Moreover, Ishii and Mitsukuri ${ }^{8)}$ discriminated 10 of the 12 rice chromosomes expected in the haploid genome of japonica, 'Norin 8', according to the both length and the arm ratio.

Concerning genome analysis, in 1939, Morinaga ${ }^{9)}$ analyzed various different rice genomes for the first time and developed the $\mathrm{A}$ to $\mathrm{E}$ genome formulas. Subsequently, in 1961, Li et al. ${ }^{10)}$ added the F genome, and in 1997, Aggarwal et al. ${ }^{11)}$ detected a further three new genomes, H, J, and K, by genomic Southern analyses. Autopolyploids and haploids, on the other hand, were found spontaneously, and in 1955, Mashima and 
Uchiyamada $^{12)}$ produced tetraploids by colchicine treatment of hybrids between japonica and indica. Niizeki and Oono ${ }^{13)}$ established an anther culture method in 1968 to produce haploid plants artificially and this technique is generally used for breeding of dihaploid lines.

With regard to linkage studies, Nishimura ${ }^{14)}$ successfully developed 12 reciprocal translocation lines of japonica rice in 1957. Furthermore, trisomic series were developed independently both in indica ${ }^{15)}$ and japonica, ${ }^{16)}$ and applied for the correspondence between chromosomes and linkage groups. ${ }^{15)-20)}$ On the other hand, saturated molecular linkage maps have been constructed by several groups ${ }^{21-24)}$ and the integration of the different linkage maps has been progressed. ${ }^{25)-27)}$ Recently the position of centromere and locations of RFLP markers of the molecular linkage maps on both arms were detected through gene dosage analysis using the secondary and telotrisomics. ${ }^{28)-30)}$ Depending on the information the orientation of the maps and allocation of genes to short and long arms were resolved. ${ }^{24), 28)-30)}$

These linkage maps were effectively used for the chromosomal identification of gene loci. In 1997, genetic linkage maps constructed on the basis of orthologous loci were compared in rice, sorghum, maize, wheat and oats, ${ }^{2)}$ revealing that gene content and order are highly conserved between different species within the grass family. Recently, physical maps and gene information have become important resources in molecular research, facilitating positional cloning of agriculturally important genes, ${ }^{31), 32)}$ sequencing of genomic DNA, ${ }^{33,34)}$ analyses of chromosome and genome structures. ${ }^{35,36)}$

Kurata and Omura ${ }^{37)}$ and $\mathrm{Wu}$ et $a l^{38)}$ previously reported the karyotypes of japonica and indica rice, respectively. The chromosomes of a variety, 'Sekitori' were arranged in order of length and designated K1 to $\mathrm{K} 12{ }^{37)}$ and the karyotypes were shown to consist of five metacentric, five submetacentric, and two subterocentric chromosome pairs. The chromosomes of indica variety 'IR36', were also studied and different karyotypes were assigned by $\mathrm{Wu}$ et $a l .{ }^{38)}$ The satellite chromosomes were shown to be K10 and K8 in japonica and indica, respectively. ${ }^{39)}$ Fukui and Iijima ${ }^{40)}$ defined, for the first time, the condensation pattern (CP) caused by uneven condensation, which appears in specifically small plant chromosomes at prometaphase. The mitotic prometaphase chromosomes in a japonica strain, 'Nipponbare', was applied to CHIAS ver. $2^{41)}$ since the CP can be effectively used to identify individual chromo- somes.

To avoid confusion using different systems, a new coordinated chromosome numbering system ${ }^{42), 43)}$ was proposed by the Rice Genetics Cooperative based on the length of pachytene chromosomes. ${ }^{44)}$ The correspondence of somatic and pachytene chromosome is further described in Fukui and Iijima ${ }^{45)}$; the chromosome numbers in this review are also based on the proposed numbering system mentioned in Khush and Kinoshita. ${ }^{43)}$

Morphological characterization of rice chromosomes at mitosis. It is generally accepted that chromosome sizes vary among plant species in correlation with genome sizes. Until recently, our understanding of rice chromosomes $(2 n=24,440 \mathrm{Mb} / \text { haploid genome })^{46)}$ was recognized as being far behind that of other plant species, especially important cereals such as maize ( $2 n=$ $10,2700 \mathrm{Mb}),{ }^{47)}$ wheat $(2 n=42,17470 \mathrm{Mb})^{48)}$ and barley $(2 n=14,5400 \mathrm{Mb}),{ }^{49)}$ because of the extremely small size.

In species with large chromosomes such as wheat, barley, and Trillium, it is possible to use typical banding patterns that appear after C- and/or N-banding treatments for chromosomal identification. ${ }^{48), 49)}$ However, in rice, it is difficult to distinguish individual chromosomes and centromere positions after pretreatments by colchicines, 8-hydroxyquinoline or cold temperature, because of the lack of banding pattern. Nevertheless, the distinct feature of rice chromosomes appears in uneven staining, that is a $\mathrm{CP}$ at mitotic prometaphase. These chromosome characteristics were analyzed using an imaging technique, providing important information that has significantly added to rice chromosome research. ${ }^{50,51)}$ Rice chromosomes at the prometaphase stage can be easily collected by simply omitting the pretreatments provided in conventional methods such as banding, and the resultant centromere positions, and the condensation patterns (CPs) have proven to be quantitatively useful in chromosome identification. ${ }^{40,50)}$ Fig. 1a shows rice chromosomes at mitotic prometaphase and the stable CP appears during this stage.

Identification of rice chromosomes by image analysis. It was previously demonstrated that the morphological characteristics of rice chromosomes can be clearly produced by computer imaging, better than with other ordinary methods. ${ }^{40)}$ The representative steps of image analysis of rice chromosomes are explained in Table I and Fig. 2. Original digitized images are initially enlarged and then a part of the chromosomal spreads (Fig. 2a) is entered onto a computer. The images are then digitally enhanced and distortion is 


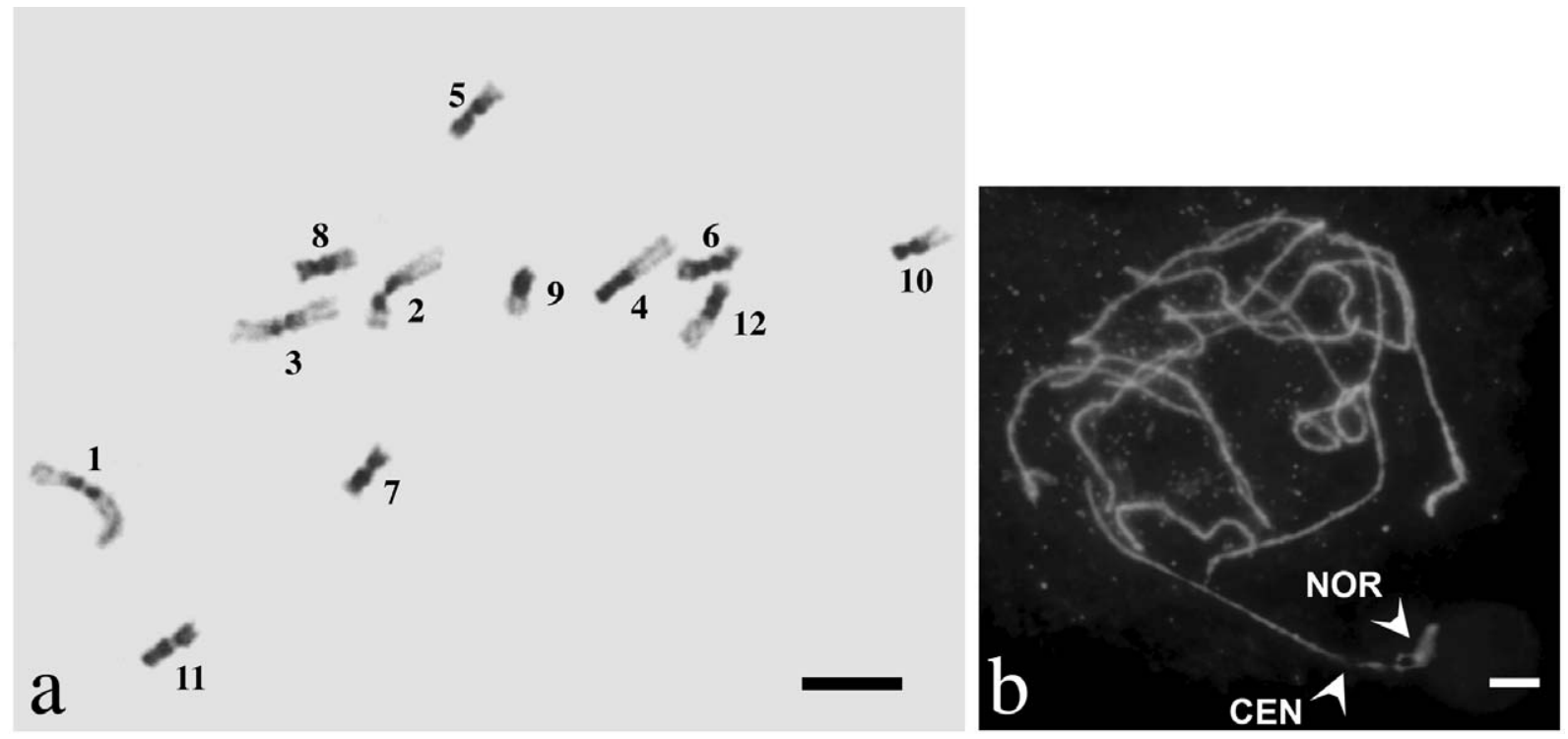

Fig. 1. Rice somatic chromosomes at prometaphase and pachytene chromosomes. a: Rice haploid chromosomes prepared according to the enzymatic maceration and air-drying (EMA) method and stained with a Giemsa solution. The scale bar $=10 \mu \mathrm{m}$. b: Pachytene chromosomes prepared according to the squash method and stained by DAPI (4', 6-diamidino-2-phenylindole) and PI (Propidium Iodide). The chromosome images of chromosome 9 are complemented with stains by DAPI and of PI. NOR region are high intense by PI (arrow). Reddish blue region indicates the NOR (nucleolar organizing region) of chromosome 9 . The arrowhead indicates centromeric regions (CEN, Centromere). The scale bar $=10 \mu \mathrm{m}$.

Table I. List of the discrimination chart ${ }^{40)}$

1. Is it possible to distinguish all the pairs of Chromosomes 4, 9 and 10 by visual inspection? Stop further procedures if not.

2. Select the each pair of Chromosomes 4, 9 and 10.

3. Select the longest three pairs of chromosomes among the remainder. Chromosomes 1,2 and 3 are selected.

4. Select a pair of the most metacentric chromosomes. They are Chromosome 3.

5. Select a pair of chromosomes with the shorter length of the short arms than another pair of the chromosomes. They are Chromosome 2.

6. Is it possible to distinguish all the pairs of Chromosomes 1,2 and 3 completely? Stop further procedures if not.

7. Select a pair of chromosomes with one and two condensed blocks on short and long arms, respectively. Chromosome 11 are identified.

8. Select a pair of chromosomes with much heavier condensation at the long arms than the short arms among the three pairs of the shortest chromosomes selected from the remaining five pairs of chromosomes. They are Chromosome 7 .

9. Select a pair of chromosomes with the shortest long arms among the remaining four pairs of chromosomes and select another pair of chromosomes with the longest short arms among the remaining three pairs of chromosomes. Then the selected two pairs of chromosomes are either a pair of Chromosome 5 or 6 .

10. Select a pair of chromosomes with the larger relative length. The longer pair is Chromosome 6 and another pair is Chromosome 5.

11. Select a pair of chromosomes with the larger difference between the condensation degrees at the proximal regions of the long and short arms. They are chromosome 12 and the remaining pair is Chromosome 8.

corrected (Fig. 2b). Following this, chromosomal regions are digitally determined and the background field is eliminated (Fig. 2c). The gray chromosome images are artificially pigmented using a look-up-table to enhance the contrast in CP visibly (Fig. 2d). As a result, detailed chromosomal structures are clearly visible than gray level presentation by 0-255 but through differences in color. Centromere positions in the enlarged image are manually determined and white lines are used to delimit the border between the long and short arms (Fig. 2e) as a centromeric region. A center (mid-rib) line is subsequently drawn on each chromatid (Fig. 2f), and a density profile 

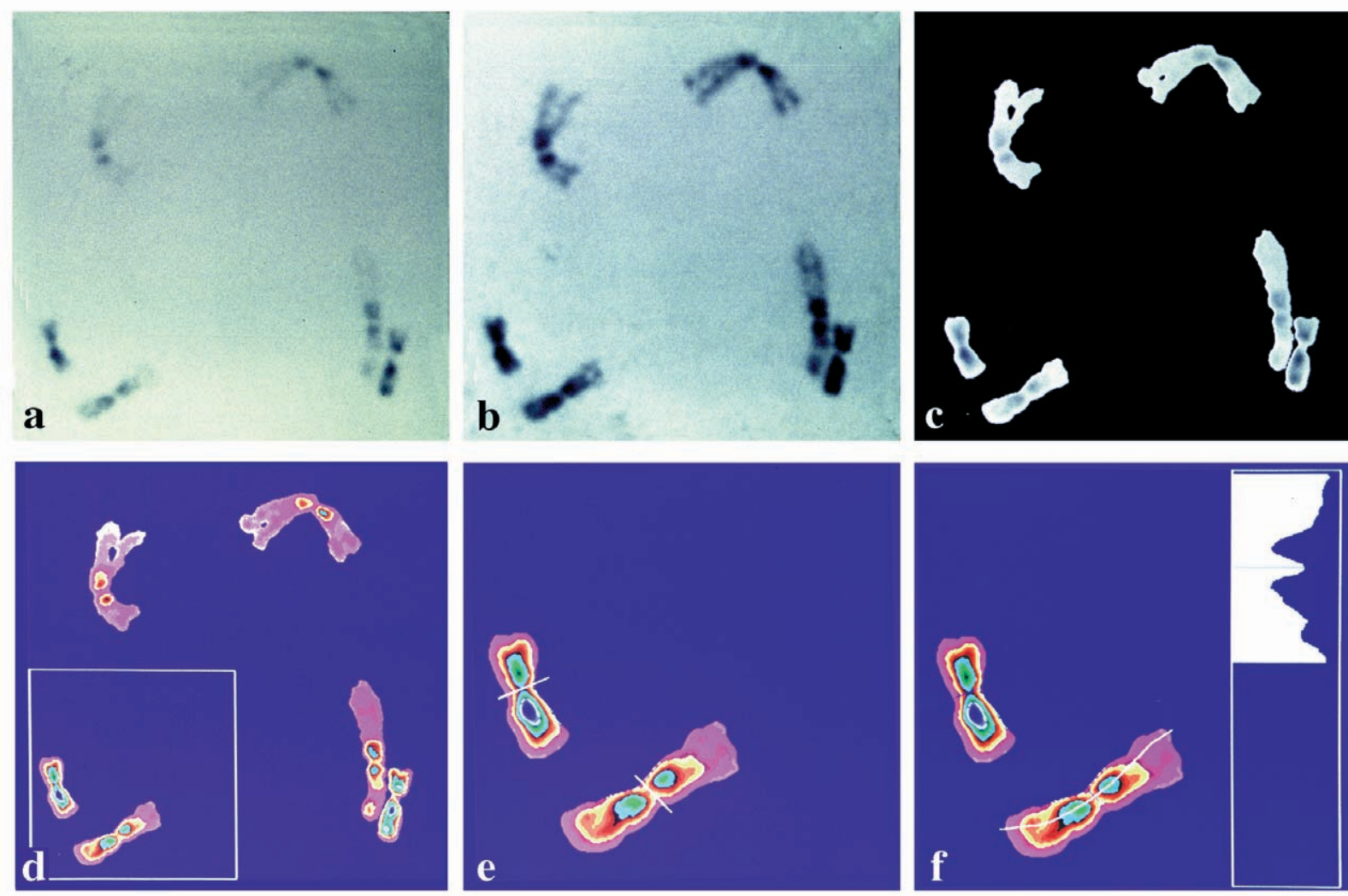

Fig. 2. Representative steps in image analysis of rice chromosomes. a: Original digitized images of part of a chromosomal spread. b: The images are digitally enhanced and distortion is corrected. c: Chromosomal regions are digitally determined and the background field is eliminated. d: Black and white chromosome images are artificially colored using a look-up-table. As a result, detailed chromosomal structures are clearly visible by differences in colors. e: Enlarged image of two chromosomes. Centromere positions are manually determined and white lines are used to delimit the border between the long and short arms. f: A center (mid-rib) line is manually drawn in each chromatid and a density profile is depicted under the line; the density profile expressed as a numbering of each arm represents a CP (condensation pattern) and is used for the development of an integrated chromosome map and automatic identification of chromosomes.

is then depicted below this.

Twelve mitotic prometaphase chromosomes are arranged in the order of chromosome number. The density profile is defined as a $\mathrm{CP}$, which is useful for constructing a quantitative map and for automatic identification of rice chromosomes. ${ }^{40), 50), 52,53)}$

Quantitative chromosome map. Using 360 chromosomes from 30 spreads at prometaphase stage, rice chromosomes were identified both visually and statistically based on their stripe patterns of CPs. Overall, $92.2 \%$ were in agreement between visual and computational methods with regard to the twelve chromosome numbers. These results demonstrated the effectiveness of CPs in identifying chromosomes and defined them as a new image parameter together with the conventional parameters of total length and arm ratio.

Average density profiles (standard CPs) based on $30 \mathrm{CPs}$ from each of the twelve rice chromosomes ${ }^{40)}$ were used for development of a quantitative idiogram (Fig. 3). The original gray image of chromosome 11 stained with a Giemsa staining solution is indicated in the bottom of Fig. 3. A centromeric (gray value $=0$ ) and center line (mid-rib line) on the chromatid are shown for the following measurement. A pseudo-colored chromosome above the gray image is a representation of the same chromosome with the white mid-rib line. The middle diagram shows the density profile at the mid-rib line; gray values 0 and 255 represent black and white, respectively, and the three concave regions correspond to the dark or condensed regions on the chromosome. The top 


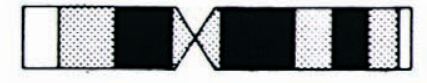

255 Grayvalue profile

$\mathbf{0}$

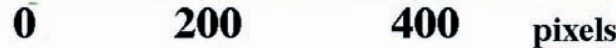
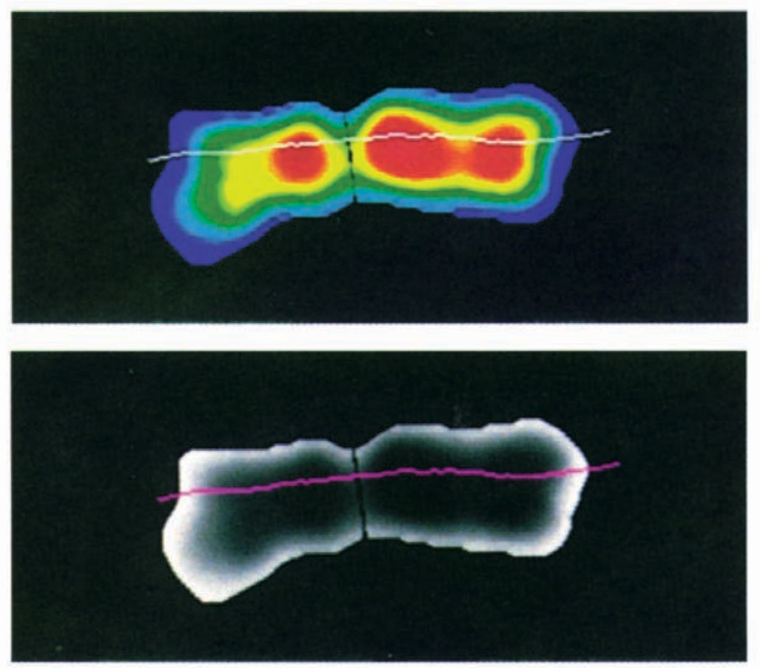

Fig. 3. Development of a quantitative idiogram based on condensation patterns (CPs). The bottom two images show the original gray image and the pseudo-colored image of chromosome 11 stained with a Giemsa solution; centromere (gray value $=0$ ) and mid-rib line (magenta or white) are included on the chromatid. The middle diagram shows the density profile at the mid-rib line. Gray values 0 and 255 represent black and white, respectively, and the three concave regions (arrows) on the CP correspond to dark or condensed regions on the chromosome. The top image shows a quantitative idiogram or quantitative chromosome map of chromosome 11. image of Fig. 3 shows a quantitative idiogram or quantitative map of the chromosome $11 .^{54)}$ Fig. 4 shows quantitative rice chromosome maps or idiograms of all 12 rice chromosomes. $^{40), 50)}$

Pachytene chromosome analysis. Shastry et $a l{ }^{55)}$ studied pachytene chromosomes and numbered them in descending order of chromosome length. In addition, Cheng et al. ${ }^{56)}$ developed an ideogram along the distribution of heterochromatin in the individual rice chromosomes based on the pattern of 4', 6-diamidino2-phenylindole (DAPI) staining of japonica pachytene chromosomes.

Pachytene chromosomes (Fig. 1b) at the meiotic stage are 10 times larger than those at the prometaphase during mitosis stage (Fig. 1a). Moreover, a significantly higher proportion of heterochromatic regions is observed in meiotic pachytene compared with somatic chromosomes, especially at centromeric regions.

Kato et $a l{ }^{57)}$ developed a method for quantitatively producing chromosome maps using pachytene chromosome 9. Computerized image analysis has not been utilized for characterization of pachytene chromosome chromomeres because their size and fluorescence intensity are differently along a chromosome. To distinguish the chromomeres along long pachytene chromosomes, gray values relative to adjacent regions were used.

Three different maps of chromosome 9, somatic chromosome, ${ }^{40)}$ pachytene chromosome ${ }^{57)}$ and saturated linkage map, ${ }^{23), 24)}$ are compared in Fig. 5 by adjusting the relative chromosome length. Eight regions (addresses) are mapped on the somatic prometaphase map and 48 on the pachytene map, and the 22 chromomeres are noted. A conspicuous difference was detected among all three maps with regard to the nucleolar organizing region (NOR) and satellite region. These results underscore the usefulness of rice chromosome maps in some plant research. There are two major benefits with quantitative chromosome maps in plant genetic research. First benefit is obvious that somatic and pachytene chromosome maps are suitable for destination genetic information on physical maps. The pachytene chromosome map is especially useful because more detail addresses can be determined compared with the prometaphase map. This higher resolution enables more accurate gene positioning with fluorescence in situ hybridization (FISH). Combining this imaging system with FISH mapping of DNA markers (e.g. anchor clones, cDNA and transgenic DNA) or repeated 


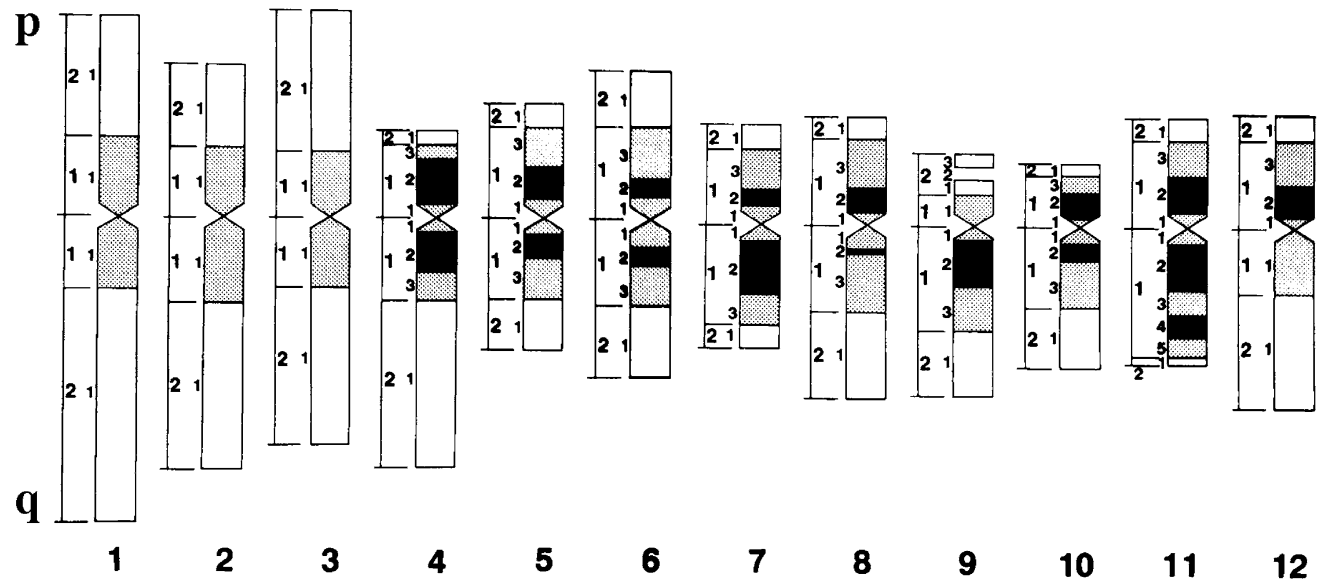

Fig. 4. Quantitative chromosome maps or idiograms of all 12 rice chromosomes. $\mathrm{p}$ and $\mathrm{q}$ indicate short and long arms, respectively. The numerical characters on the left of each chromosome indicate the address of chromosomal positions. $P i$ - $b$ gene is addressed on $2 \mathrm{q} 2.1$. The nucleolar organizing region (NOR) is located at $9 \mathrm{p} 2.2$.
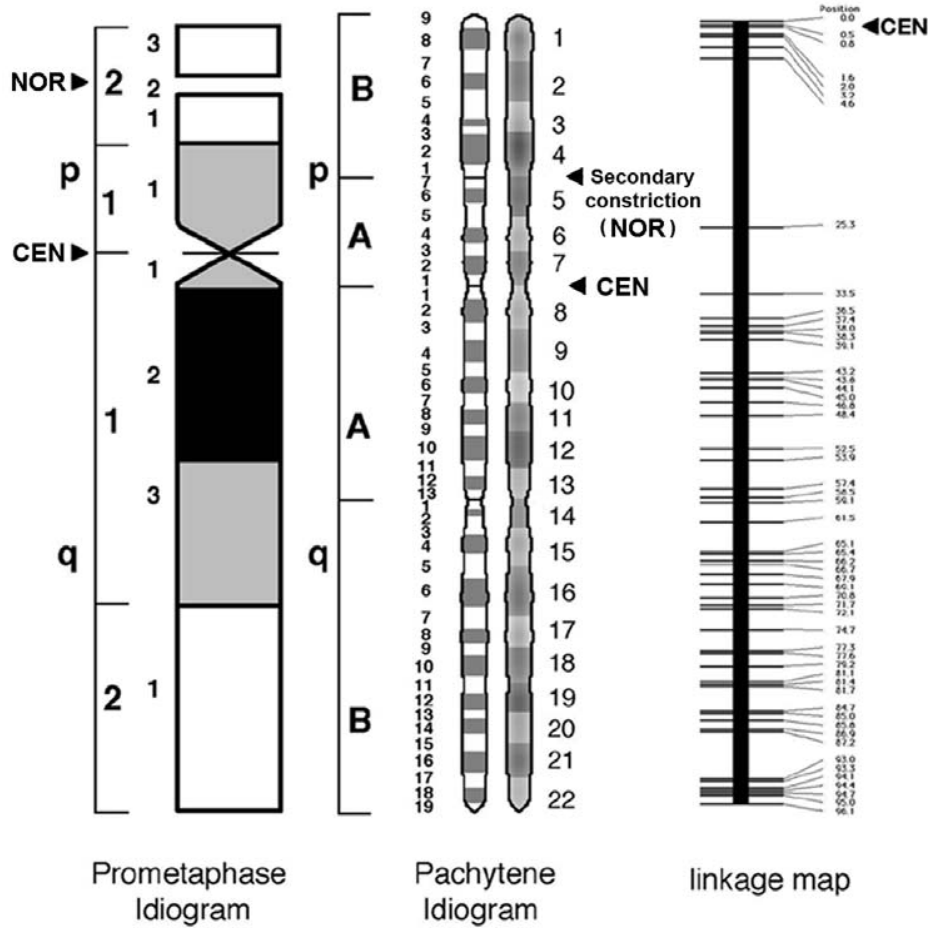

\section{Pachytene Idiogram}

linkage map

\section{Chromosome 9}

Fig. 5. Three different maps of rice chromosome 9. Somatic chromosome, pachytene chromosome and molecular linkage maps (quoted from Ref. 40, 57 and 24, respectively.) were adjusted according to chromosome length. Eight regions (addresses) are mapped on the somatic prometaphase map, and the 22 chromomeres marked on the pachytene map (Index ideogram). Conspicuous differences at the secondary constriction, nucleolar organizing region (NOR) and centromere (CEN) were recognized among the three maps. 


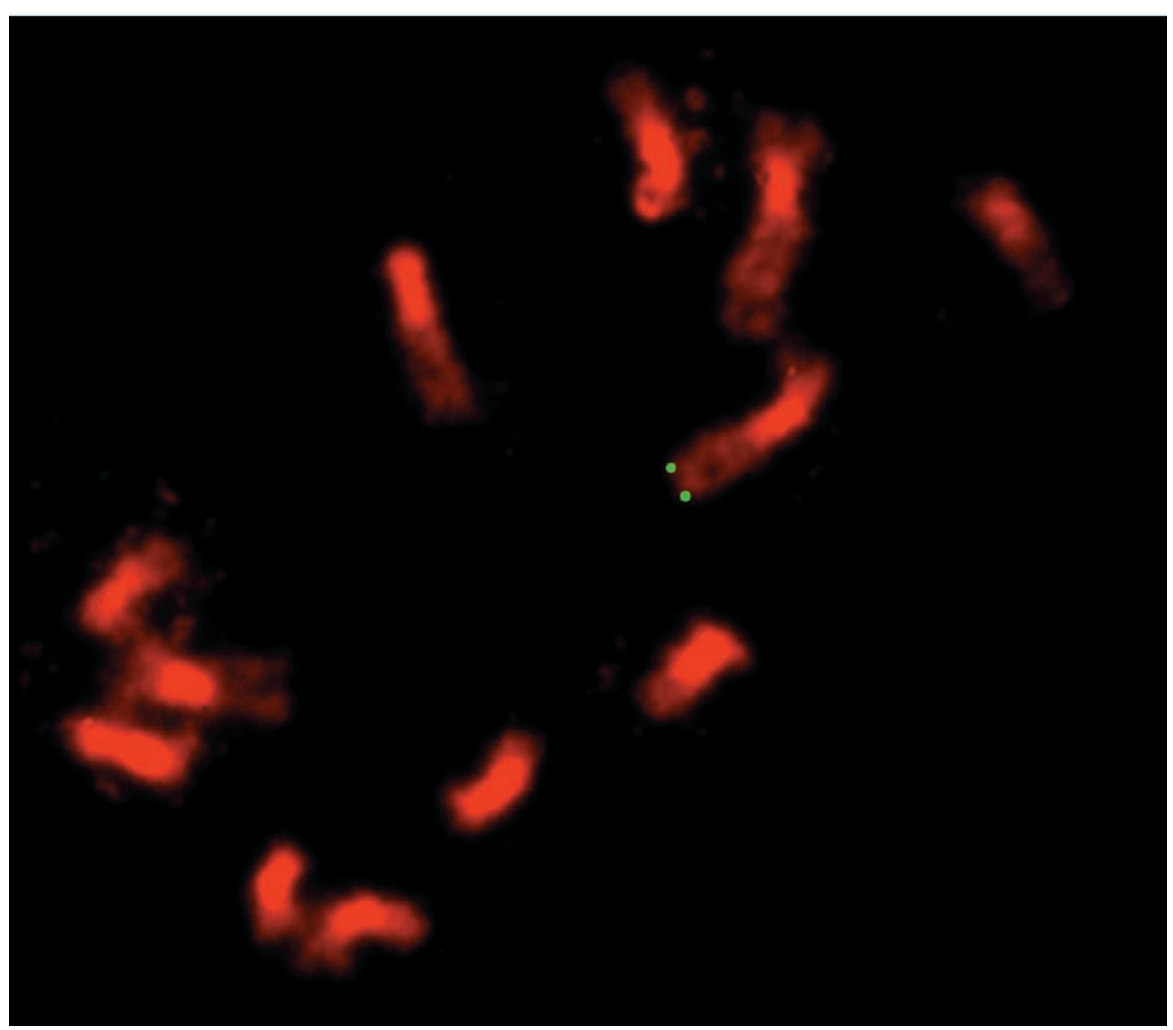

Fig. 6. FISH image of the BAC clone containing the leaf blast resistant gene (Pib, green signals) physically located on rice chromosome 2 .

sequences would allow precise physical positioning of rice chromosomes. ${ }^{58)-60)}$

As second benefit, densely located chromomeres serve as land markers for precise positioning of structurally specific regions, such as heterochromatic and euchromatic regions. Morphological characteristics of pachytene and prometaphase chromosomes will also serve as clues in rice chromosome identification and analysis of higher structures such as centromeres and telomeres. In so-called genetic linkage maps, two loci are measured by the degree of recombination frequency and expressed as a percentage or centi-Morgan, which is postulated on the basis that the recombinations occur homogeneously on all chromosomes. ${ }^{61), 62)}$ However, according to various cytological findings, several uneven structures are found in different parts of the chromosomes. ${ }^{63), 64)}$ It is known that centromere regions usually inhibit the recombination. An overall view of recombination frequency per unit length of chromosome can be attained by combining the physical positioning of markers with a molecular linkage map. ${ }^{65)}$

Visual verification of the rice blast resis- tance gene. Fig. 6 shows the BAC clones used as probes in FISH experiments for evaluating the detection sensitivity of fluorescent signals on rice chromosomes. The DNA clones were visualized after FISH due to their fluorescence signal. ${ }^{66)}$ Rice blast is the most important fungal disease caused by Magnaporthe grisea (Hebert) Barr. The one of the leaf blast resistant genes Pib, was cloned into a BAC clone with a $180-\mathrm{kb}$ insert of rice genomic DNA. Because the location of the genomic DNA on rice chromosomes is unknown, the FISH method was employed to physically determine the actual location of the BAC clone and thus the position of Pib. Fig. 6 shows the signals from the BAC clone at the distal end of the long arm of chromosome $2 .{ }^{67)}$ A haploid rice plant derived by an anther culture was used for chromosome preparations. Doublet signals are crucial for discriminating between genuine signals and noise. According to the rice chromosome map, the chromosomal location of the $P i b$ gene was consequently addressed on 2q2.1 (Fig. 4). Quantitative chromosome maps therefore contain important biological information for analysis of gene function and structure, and thus play 
Table II. Nuclear DNA content of the 12 diploid rice species determined by flow cytometry ${ }^{46)}$

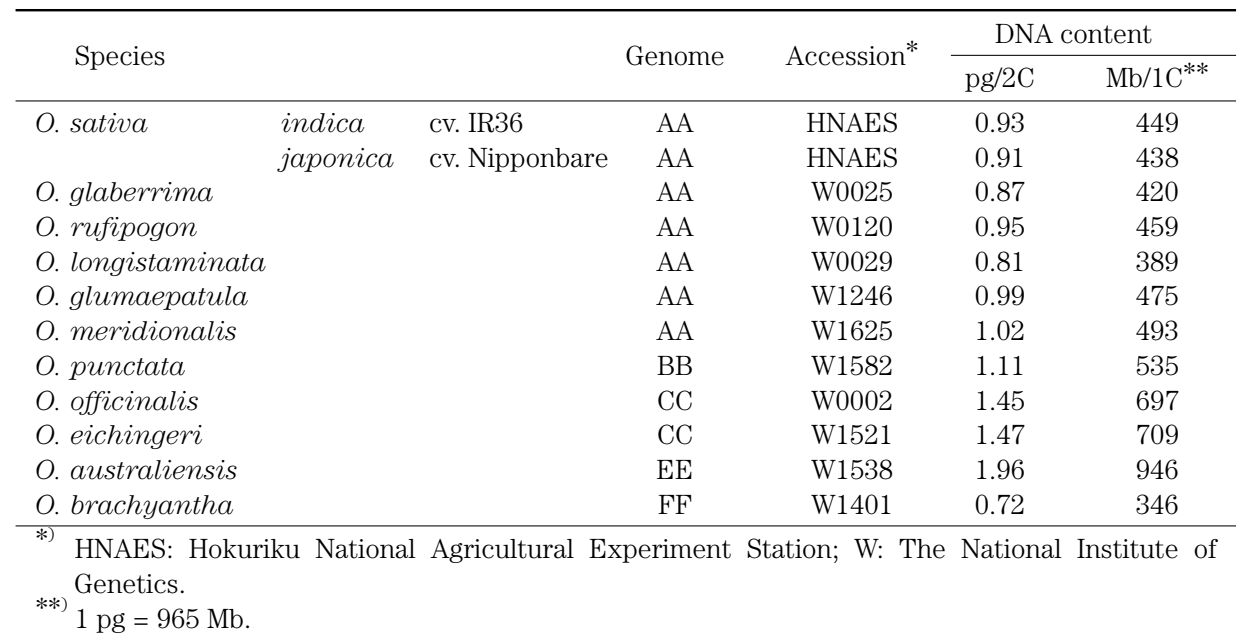

an important role in rice genetics and breeding. ${ }^{68)}$

Rice chromosomes in different genomes. The nuclear DNA content and genome size of Oryza species have been determined using different methods of microphotodensitometry ${ }^{69), 70)}$ and flow cytometry. ${ }^{71)}$ Estimated genome sizes are shown to vary among Oryza species from 392 to $1142 \mathrm{Mb}$. Although there is a considerable variation among plural data, allotetraploid species (e.g. CCDD and BBCC) tend to have more nuclear DNA contents than diploid species. In addition, B and $\mathrm{C}$ genome species have more nuclear DNA than A and $\mathrm{F}$ species. It was previously noted that $O$. australiensis, which has an $\mathrm{E}$ genome, has the largest genome of all diploid species. The precise genome sizes of diploid species in the genus Oryza were therefore examined using the same flow cytometry with two internal controls of chicken red blood cells (CRBC) and O. sativa cv. Nipponbare cells, respectively, as shown in Table II. ${ }^{46}$ An approximately 2.7-fold variation in nuclear DNA content ranging from $0.72 \mathrm{pg} / 2 \mathrm{C}$ in $O$. brachyantha to $1.96 \mathrm{pg} / 2 \mathrm{C}$ in $O$. australiensis was observed.

According to genome size and DNA content among chromosomes of eight diploid rice species, $O$. australiensis chromosomes are much larger than those of all other diploid species and proximal regions show dense heterochromatic condensation. On the other hand, the chromosomes of $O$. brachyantha are the smallest and visible heterochromatic regions are quite limited. In statistical analyses, correlation coefficient between the total chromosome lengths and nuclear
DNA contents was 0.939 and significant at the $0.1 \%$ level. Thus, chromosome length seems to be a good indicator of rice genome size in Oryza species.

Conclusions. Rice genome research has therefore entered a new era. New cytogenetic technology combined with computer and molecular approaches has enabled somatic and pachytene chromosome karyotyping, genome analysis, precise measurement of genomic regions among rice species using flow cytometry, gene mapping by FISH, and computer image analysis. It is anticipated that the results of such basic rice chromosome research will provide indispensable information for rice genetics and breeding in various fields of study. Thus, the development of rice chromosome map contributes to determine precise contents showing recombination and translocation on rice chromosomes.

By applying the new basic information obtained from conventional and molecular linkage mappings together with quantitative chromosome maps, many efficient and reliable rice researches are likely to be developed in the near future. ${ }^{61), 62), 72)}$

\section{References}

1) Sasaki, T., and Burr, B. (2000) International rice genome sequencing project: the effort to completely sequence the rice genome. Curr. Opin. Plant Biol. 3, 138-141.

2) Devos, K. M., and Gale, M. D. (1997) Comparative genetics in the grasses. Plant Mol. Biol. 35, 3-15.

3) Kuwada, Y. (1910) A cytological study of Oryza sativa L. Bot. Mag. 24, 267-280.

4) Rau, N. S. (1929) Further contributions to the cytology of 
some crop plants of south India. J. Indian Bot. Soc. 81, 201-206.

5) Nandi, H. K. (1936) The chromosome morphology, secondary association and the origin of cultivated rice. J. Genet. 33, 315-336.

6) Yasui, K. (1941) Diploid bud formation in a haploid Oryza with some remarks on the behaviour of nucleolus in mitosis. Cytologia 11, 515-525.

7) Hu, C. H. (1964) Further studies on the chromosome morphology of Oryza sativa. I. In Rice Genetics and Cytogenetics, Proc. Sym. on Rice Genetics and Cytogenetics. Elsevier Publishing Co., Amsterdam, pp. 51-61.

8) Ishii, K., and Mitsukuri, S. (1960) Chromosome studies on Oryza. I. Somatic chromosomes of Oryza sativa L. Bull. Res. Coll. Agric. Vet. Med. Nihon Univ. 11, 44-53.

9) Morinaga, T. (1939) Cytogenetics on rice (Oryza sativa L.). Bot. \& Zool. 7, 179-183.

10) Li, H. W., Weng, T. S., Chen, C. C., and Wang, W. H. (1961) Cytogenetical studies of Oryza sativa L. and its related species. I. Hybrids $O$. paraguaiensis Wedd. $\times$ O. brachyantha Chev. et Roehr., O. paraguaiensis Wedd. $\times$ O. australiensis Domin and O. australiensis Domin $\times$ O. alta Swallen. Bot. Bull. Acad. Sinica 2, 79-86.

11) Aggarwal, R. K., Brar, D. S., and Khush, G. S. (1997) Two new genomes in the Oryza complex identified on the basis of molecular divergence analysis using total genomic DNA hybridization. Mol. Gen. Genet. 254, 1-12.

12) Mashima, I., and Uchiyamada, H. (1955) Studies on the breeding of fertile tetraploid plants of rice. Bull. Nat. Inst. Agr. Sci. Japan D 5, 104-136.

13) Niizeki, H., and Oono, K. (1968) Induction of haploid rice plant from anther culture. Proc. Japan Acad. 44, 554-557.

14) Nishimura, Y. (1957) Studies on the reciprocal translocation in rice and barley. Bull Natl. Inst. Agr. Sci., Ser. D 9, 171-235.

15) Khush, G. S., Singh, R. J., Sur, S. C., and Librojo, A. L. (1984) Primary trisomics of rice: Origin, morphology, cytology, and use in linkage mapping. Genetics 107, 141-163.

16) Iwata, N., and Omura, T. (1984) Studies on the trisomics in rice plants (Oryza sativa L.). VI. An accomplishment of trisomic series in japonica rice plants. Jpn. J. Genet. 59, 199-204.

17) Iwata, N., and Omura, T. (1971) Linkage analysis by reciprocal translocation method in rice plants (Oryza sativa L.). I. Linkage groups corresponding to the chromosomes 1, 2, 3 and 4. Japan. J. Breed. 21, 19-28.

18) Iwata, N., and Omura, T. (1971) Linkage analysis by reciprocal translocation method in rice plants (Oryza sativa L.). II. Linkage groups corresponding to the chromosomes 5, 6, 8, 9, 10 and 11. Sci. Bull. Fac. Agr. Kyushu Univ. 25, 137-153.

19) Sato, S., Kinoshita, T., and Takahashi, M. (1973) Linkage analysis of rice plant, by the use of Nishimura's reciprocaltranslocation lines. - Genetical studies on rice plant, LIV -. Mem. Fac. Agr. Hokkaido Univ. 8, 367-376.

20) Yoshimura, A., Ideta, O., and Iwata, N. (1982) Linkage analy- sis by reciprocal translocation method in rice plants (Oryza sativa L.). III. Marker genes located on chromosomes 2, 3, 4 and 7. Japan. J. Breed. 32, 323-332.

21) Saito, A., Yano, M., Kishimoto, N., Nakagahra, M., Yoshimura, A., Saito, K., Kuhara, S., Ukai, Y., Kawase, M., Nagamine, T. et al. (1991) Linkage map of restriction fragment length polymorpholism loci in rice. Japan. J. Breed. 41, 665-670.

22) Causse, M. A., Fulton, T. M., Cho, Y. G., Ahn, S. N., Chunwongse, J., Wu, K., Xiao, J., Yu, Z., Ronald, P. C., Harrington, S. E. et al. (1994) Saturated molecular map of the rice genome based on an interspecific backcross population. Genetics 138, 1251-1274.

23) Kurata, N., Nagamura, Y., Yamamoto, K., Harushima, Y., Sue, N., Wu, J., Antonio, B. A., Shomura, A., Shimizu, T., Lin, S. Y. et al. (1994) A 300 kilobase interval genetic map of rice including 883 expressed sequences. Nature Genet. 8, 365-372.

24) Harushima, Y., Yano, M., Shomura, A., Sato, M., Shimano, T., Kuboki, Y., Yamamoto, T., Lin, S. Y., Antonio, B. A., Parco, A. et al. (1998) A highly-density rice genetic linkage map with 2275 markers using a single F2 population. Genetics 148, 479-494.

25) Kishimoto, N., Foolad, M. R., Shimosaka, E., Matsuura, S., and Saito, A. (1993) Alignment of molecular and classical linkage maps of rice, Oryza sativa. Plant Cell Reports 12, 457-461.

26) Xiao, J., Fulton, T., McCouch, S. R., Tanksley, S. D., Kishimoto, N., Ohsawa, R., Ukai, Y., and Saito, A. (1992) Progress in integration of the molecular maps of rice. Rice Genet. Newslett. 9, 124-128.

27) Yoshimura, A., Ideta, O., and Iwata, N. (1997) Linkage map of phenotype and RFLP markers in rice. Plant Mol. Biol. 35, 49-60.

28) Singh, K., Multani, D. S., and Khush, G. S. (1996) Secondary trisomics and telotrisomics of rice: Origin, characterization and use in determining the orientation of chromosome map. Genetics 143, 517-529.

29) Singh, K., Ishii, T., Parco, A., Huang, N., Brar, D. S., and Khush, G. S. (1996) Centromere mapping and orientation of molecular linkage map of rice (Oryza sativa L.). Proc. Natl. Acad. Sci. USA 93, 6163-6168.

30) Khush, G. S., Singh, K., Ishii, T., Parco, A., Huang, N., Brar, D. S., and Multani, D. S. (1996) Centromere mapping and orientation of the cytological, classical and molecular linkage maps of rice. In Rice Genetics III (ed. Khush, G. S.). IRRI, Manila, Philippines, pp. 57-75.

31) Wang, G. L., Ruan, D.-L., Song, W.-Y., Sideris, S., Chen, L., Pi, L.-Y., Zhang, S., Zhang, Z., Fauquet, C., Gaut, B. S. et al. (1998) Xa21D encodes a receptor-like molecule with a leucine-rich repeat domain that determines race-specific recognition and is subjected to adaptive evolution. Plant Cell 10, 765-780.

32) Akagi, H., Nakamura, A., Yokozeki-Misono, Y., Inagaki, A., Takahashi, H., Mori, K., and Fujiyama, T. (2004) Positional cloning of the rice RF-1 gene, a restorer of BT-type cytoplasmic male sterility that encodes a mitochondria-targeting 
PPR protein. Theor. Appl. Genet. 108, 1449-1457.

33) Sasaki, T., Matsumoto, T., Yamamoto, K., Sakata, K., Baba, T., Katayose, Y., Wu, J., Niimura, Y., Cheng, Z., Nagamura, Y. et $a l$. (2002) The genome sequence and structure of rice chromosome 1. Nature 420, 312-316.

34) Feng, Q., Zhang, Y., Hao, P., Wang, S., Fu, G., Huang, Y., Li, Y., Zhu, J., Liu, Y., Hu, X. et al. (2002) Sequence and analysis of rice chromosome 4. Nature 420, 316-320.

35) Blair, M. W., Hedetale, V., and McCouch, S. R. (2002) Fluorescent-labeled microsatellite panels useful for detecting allelic diversity in cultivated rice (Oryza sativa L.). Theor. Appl. Genet. 105, 449-457.

36) Coburn, J. R., Temnykh, S. V., Paul, E. M., and McCouch, S. R. (2002) Design and application of microsatellite marker panels for semiautomated genotyping of rice (Oryza sativa L.). Crop Sci. 42, 2092-2099.

37) Kurata, N., and Omura, T. (1978) Karyotype analysis in rice. I. A new method for identifying all chromosome pairs. Jpn. J. Genet. 53, 251-255.

38) Wu, H. K., Chung, M. C., and Chen, M. H. (1985) Karyotype analysis of cultivar IR36. Rice Genet. Newslett. 2, 54-57.

39) Fukui, K., and Mukai, Y. (1988) Condensation pattern as a new image parameter for the identification of small chromosomes in plants. Jpn. J. Genet. 63, 359-366.

40) Fukui, K., and Iijima, K. (1991) Somatic chromosome map of rice by imaging methods. Theor. Appl. Genet. 81, 589-596.

41) Fukui, K. (1986) Comparison of the Giemsa and orcein staining methods in rice chromosomes. La Kromosomo II43-44. 1398-1404.

42) Khush, G. S. (1990) Report of meetings to discuss chromosome numbering system in rice. Rice Genet. Newslett. 7, 12-15.

43) Khush, G. S., and Kinoshita, T. (1991) Rice karyotype, marker genes, and linkage groups. In Rice Biotechnology (eds. Khush, G. S. and Toennissen, G. H.). IRRI, C.A.B. International Wallingford, U.K., pp. 83-108.

44) Wu, H. K., and Chung, M. C. (1988) Identification of extra chromosomes of six IR36 Triplo lines. Rice Genet. Newslett. 5, 41-46.

45) Fukui, K., and Iijima, K. (1992) Manual on rice chromosomes. 2nd ed., Misc. Pub. Natl. Inst. Agrobiol. Resour. 4, 1-25.

46) Uozu, S., Ikehashi, H., Ohmido, N., Ohtsubo, H., Ohtsubo, E., and Fukui, K. (1997) Repetitive sequences: cause for variation in genome size and chromosome morphology in the genus Oryza. Plant Mol. Biol. 35, 791-799.

47) Bennett, M. D., and Leitch, I. J. (1995) Nuclear DNA amounts in Angiosperms. Ann. Botany 76, 113-176.

48) Endo, T. R. (1986) Complete identification of wheat chromosomes by means of a C-banding technique. Jpn. J. Genet. 61, 89-93.

49) Kakeda, K., Fukui, K., and Yamagata, H. (1991) Heterochromatic differentiation in barley chromosomes revealed by $\mathrm{C}-$ and N-banding techniques. Theor. Appl. Genet. 81, 144-150.

50) Fukui, K. (1996) Advances in rice chromosome research,
1990-95. In Rice Genetics III. Proc. 3rd Intl. Rice Genet. Symp. (ed. Khush, G. S.). Intl. Rice Res. Inst., Manila, Philippines, pp. 117-130.

51) Fukui, K., and Ohmido, N. (1999) An alternative approach based on molecular cytology. In Genomes (ed. Gustafson, J. P.). Plenum Press, New York, pp. 109-121.

52) Kamisugi, Y., and Fukui, K. (1990) Automatic karyotyping of plant chromosomes by imaging techniques. BioTechnique $\mathbf{8}$, 290-295.

53) Fukui, K. (1996) Plant chromosomes at mitosis. In Plant Chromosomes: Laboratory Methods (eds. Fukui, K., and Nakayama, S.). CRC Press, New York, pp. 1-18.

54) Kamisugi, Y., Furuya, N., Iijima, K., and Fukui, K. (1993) Computer-aided automatic identification of rice chromosomes by image parameters. Chromosome Res. 1, 189-196.

55) Shastry, S. V., Ranga, S., Rao D. R., and Misra, R. N. (1960) Pachytene analysis in Oryza. I. Chromosome morphology in Oryza sativa. Indian J. Genet. Plant Breed. 20, 15-21.

56) Cheng, Z., Buell, C. R., Wing, R. A., Gu, M., and Jiang, J. (2001) Toward a cytological characterization of the rice genome. Genome Res. 11, 2133-2141.

57) Kato, S., Ohmido, N., and Fukui, K. (2003) Development of a quantitative pachytene chromosome map in Oryza sativa by imaging methods. Genes Genet. Syst. 78, 155-161.

58) Jiang, J., Gill, B. S., Wang, G. L., Ronald, P. C., and Ward, D. C. (1995) Metaphase and interphase fluorescence in situ hybridization mapping of the rice genome with bacterial artificial chromosomes. Proc. Natl. Acad. Sci. USA 92, 4487-4491.

59) Cheng, Z., Buell, C. R., Wing, R. A., and Jiang, J. (2002) Resolution of fluorescence in-situ hybridization mapping on rice mitotic prometaphase chromosomes, meiotic pachytene chromosomes and extended DNA fibers. Chromosome Res. 10, 379-387.

60) Kumekawa, N., Ohmido, N., Fukui, K., Ohtsubo, E., and Ohtsubo, H. (2001) A new gypsy-type retrotransposon, RIRE7: preferential insertion into the tandem repeat sequence $\mathrm{TrsD}$ in pericentromeric heterochromatin regions of rice chromosomes. Mol. Genet. Genomics $\mathbf{2 6 5}$, 480-488.

61) Kinoshita, T. (1998) Linkage mapping using mutant genes in rice. Rice Genet. Newslett. 15, 13-74.

62) Kinoshita, T. (2005) Chapter 12. Linkage mapping using mutant genes in rice. In Role of classical mutation breeding in crop improvement (ed. Datta, S. K.). Daya Publishing House, Delhi, pp. 201-259.

63) Fukui, K., and Kakeda, K. (1990) Quantitative karyotyping of barley chromosomes by image analysis methods. Genome 33, 450-458.

64) Gustafson, J. P., Butler, E., and McIntyre, C. L. (1990) Physical mapping of a low-copy DNA sequence in rye (Secale cereale L.). Proc. Natl. Acad. Sci. USA 87, 1899-1902.

65) Künzel, G., Korzun, L., and Meister, A. (2000) Cytologically integrated physical RFLP maps for the barley genome based on 
translocation breakpoints. Genetics 154, 397-412.

66) Ohmido, N., Akiyama, Y., and Fukui, K. (1998) Physical mapping of unique nucleotide sequences on identified rice chromosomes. Plant Mol. Biol. 38, 1043-1052.

67) Nakamura, S., Asakawa, S., Ohmido, N., Fukui, K., Shimizu, N., and Kawasaki, S. (1997) Constitution of an 800-kb contig in the near-centromeric region of the rice blast resistance gene $P i-t a^{2}$ using a highly representative rice BAC library. Mol. Gen. Genet. 254, 611-620.

68) Ohmido, N., and Fukui, K. (2004) Recent advances in FISH analysis of plant chromosomes. Recent Res. Devel. Biochem. 5, 267-279.

69) Katayama, T. (1971) Radiosensitivity in plants. II. Relationships between radiosensitivity and DNA content per nucleus of diploid species in Oryza. Japan. J. Breed. 21, 241-246.
70) Katayama, T. (1972) Radiosensitivity in plants. III. Relationships between radiosensitivity and DNA content per nucleus of polyploid plants in Oryza. Japan. J. Breed. 22, 153-158.

71) Arumurganathan, K., and Earle, E. D. (1991) Nuclear DNA content of some important plant species. Plant Mol. Biol. Rep. 9, 208-218.

72) Kinoshita, T., and Ishikawa, R. (2002) Genetical studies of isoenzymes in rice plants I. - General viewpoint -. Recent Res. Devel. Genet. 2, 43-95.

(Received Oct. 3, 2005; accepted Nov. 14, 2005)

\section{Profile}

Dr. Toshiro Kinoshita was born in 1931 in Sapporo. After graduating from the Faculty of Agriculture, Hokkaido University in 1958, he was appointed as Instructor there. He was promoted to Professor of Plant Breeding in 1981 and has devoted himself to education and research work in plant genetics and breeding for over 40 years. After his retirement from the University, he was elected Professor Emeritus of Hokkaido University in 1994. He also served as a member of the Japanese Science Council during 1991 and 1997. His doctorial thesis published in 1970, 'Genetical studies of the male sterility of sugar beet (Beta vulgaris L.) and its related species' was highly evaluated by the related scientists. In this report he demonstrated a cytoplasmic mutation of the male sterility induced by gamma ray irradiation, the first time in the world. He extended this study to the interaction between nuclear genes and cyto-

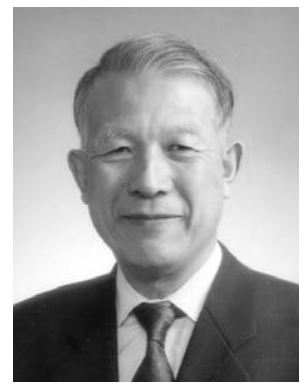
plasmic factors at both plant and molecular levels in sugar beet, rice and wheat. He was awarded the Japan Academy Prize for this research in 1993. He also succeeded to the research projects of 'Genetical studies on rice plant' from the preceding professors, Dr. S. Nagao and Dr. M. Takahashi, and developed extensive research works from the fundamental genetics to new breeding science including cellular and molecular genetics in cooperation with his co-workers. In connection with the Rice Genetics Cooperative which was established in 1985 to promote international cooperation in rice genetics, he served as a convener in the Committee on gene symbolization, nomenclature and linkage groups until 1990 and served as Chairman during 1990 and 2000. He promoted the exchange of information through the Rice Genetics Newsletter and the International Rice Genetics Symposium among the rice scientists in the world. In recognition of his brilliant achievements he has been awarded the following honors in various fields. Award of Breeding Science from the Japanese Society of Breeding in 1977; Award of Science and Technology from the Hokkaido Prefecture in 1991; Order of the Rising Sun, Third Class from the Japanese Government in 2002; Honorary Fellow of the Japanese Society of Breeding in 2004. 